\title{
Array Pattern Synthesis Using a Digital Position Shift Method
}

\author{
Chuang HAN, Ling WANG \\ School of Electronics and Information, Northwestern Polytechnical University, 710072 Xi'an, Shannxi, China \\ hanchuang357@163.com, lingwang@nwpu.edu.cn \\ Manuscript received February 15, 2016
}

\begin{abstract}
Considering all possible steering directions for beam scanning, a digital position shift method (DPSM) is presented to minimize the Peak Sidelobe Level (PSL) by searching the best position solution for every sensor and calculating the pattern with position offset factor. For the truly minimum PSL, digital position shift with optimal amplitude (DPSOA) is considered simultaneously for beam scanning. For searching the best solution to the two methods, constrained conditions for position shift range and amplitude range are described. The method of feedback particle swarm optimization (FPSO) is presented to obtain a large searching space and fast convergence in local space with refined solution. Numerical examples show that the optimized results by DPSM and DPSOA in all steering directions can be used in beam scanning for its digital realization. When compared with the other techniques published in the literature, especially the steering direction close to endfire direction, this method has lower PSL when the main beam width is maintained.
\end{abstract}

\section{Keywords}

Digital position shift method (DSPM), feedback particle swarm optimization (FPSO), beam scanning, Peak Sidelobe Level (PSL)

\section{Introduction}

Many methods can be adopted for array pattern synthesis of minimum Peak Sidelobe Level (PSL) [1]-[4]. These methods are proposed for nonlinear arrays, alternatively termed as aperiodic arrays, to search a sensors position distribution solution and they can use less number of sensors to meet similar pattern specifications [5]-[8]. Aperiodic arrays with optimally spaced sensors have the advantage of achieving higher spatial resolutions or lower sidelobe. On the other hand, many techniques proposed in the literatures adjust the weight coefficients when deriving the solution to beam pattern synthesis [9]-[11]. Phase-only control widely used in phased arrays to provide beam scanning are less expensive to produce, and also, are more likely to minimize excitation errors and preserve coherence [10].

After considering the above-mentioned aspects, a Simulated Annealing (SA)-based method was proposed in [15] to design an asymmetric array by optimizing both the sensor positions and array complex weight coefficients. It does not simultaneously optimize all the parameters, but perturbs the weight coefficient and position of each sensor in turns. In addition, it searches the sensor positions over a grid space. Although the SA based method [15] has high performance in array pattern synthesis, it is possible that sparse arrays with continuously spaced sensors could have a high degree of freedom in lowering the PSL [13].

Although many studies have been published on aperiodic array pattern synthesis, we should notice that the PSL sharply decreases with the steering angle scanning from normal direction to the edge direction when keeping the mainbeam width unchanged [13]. In [13], the authors proposed that when the array steering direction is close to the endfire direction of the array, the PSL will be very high, because the effective array aperture is very small.

In more important applications, we want to realize beam steering scanning considering all possible angles [12], but [13] presented that the PSL of beam scanning is higher than that of a specific steering direction for the limit of the minimum PSL close to endfire direction. If we can realize the properties of aperiodic array using digital methods and lower the PSL of endfire direction steering, the performance of normal direction will be retained without effect of above consider for the independent of every angles. In digital realization, we can combine aperiodic distribution and weight coefficients for lower PSL with respect to beam scanning.

Further, there are virtual array synthesis methods for planar array [14]. These methods are similar in some ways to several virtual uniform array factors of linearly weighted superposition instead of the actual array factor, and their optimized objective function works to minimize the integral of the squared absolute values between the expected and the designed array factors [10], [14]. The optimal parameter of sensor number may not be one and the weights 
of designed array is calculated, not the position of every element.

The challenge of determining optimum parameter values simultaneously stems from the nonlinear and nonconvex dependency of the array factor on the weights and the sensor positions [16]. The performance of the employed optimization scheme is an important factor in the success of a pattern synthesis method, in terms of solution quality, computational load, and stability. Particle swarm optimization (PSO) has received considerable attention because of its simplicity of implementation and its capability of escaping from the traps of local optima [17], [18].

Significant amount of research has been carried out to address the main limitation of the PSO, which is its tendency to converge prematurely at the local optima [19]. In [18], the authors revealed that one of the aspects of PSO's capability of finding the global optima mainly depends upon the capability of exploring the search space. Initial higher value of inertia weight applied to the last velocity improves exploration of the search space and its lower value toward the end of search helps to attain faster convergence [18].

Considering all possible steering directions for beam scanning, a digital position shift method (DPSM) is presented to minimize the PSL by searching the best position solution for every sensor and calculating the pattern with position offset factor. For the truly minimum sidelobe level, digital position shift and optimal amplitude (DPSOA) are both considered simultaneously for beam scanning. For searching the best solution to the above two methods, the feedback particle swarm optimization (FPSO) is presented for a large initial searching space as well as a fast convergence in local space with refined solution. The optimized results by DPSM and DPSOA in all steering directions can be used in beam scanning for its digital realization, which has lower PSL with maintaining main beam width than the other techniques published in the literature, especially the steering direction close to endfire direction.

The remaining part of this paper is organized as follows. In Sec. 2, the DPSM and DPSOA are presented for linear array. The signal forms of DPSM in applications and constrained conditions for position shift range are described, and FPSO is presented in Sec. 3. Section 4 simulates numerical examples with considering beam scanning and describes the comparative performance of the presented technique. Concluding remarks are given in Sec. 5.

\section{DPSM on Array Pattern Synthesis}

Consider a uniformly planar array of $M$ isotropic elements with equal spacing of $d$ as shown in Fig. 1 (solid). The position of each element along with $\mathrm{x}$-axis can be written as $x_{i}$. We only consider the array factor, which can be expressed as

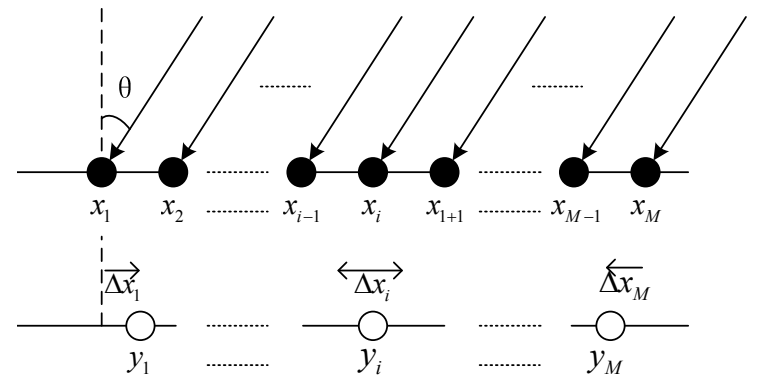

Fig. 1. The virtual array with position shift from actual array.

$$
f_{\mathrm{A}}(u)=\sum_{i=1}^{M} w_{i} \exp \left(j 2 \pi x_{i} u / \lambda\right)
$$

where $u=\sin (\theta), \theta$ (measured from $z$-axis) is the pitch angle of radiation for transmit array and the incidence of the plane wave for receive array. $\lambda$ is the wavelength, and $w_{i}$ is the weight coefficient of the $i$ th sensor. Because $w_{i}$ is complex, it can be expressed as $w_{i}=\beta_{i} \exp \left(j \phi_{i}\right)$, where $\beta_{i}$ and $\phi_{i}$ are the amplitude and phase of $w_{i}$ respectively.

We can write the response of the $i$ th actual element as

$$
A_{i}(u)=\exp \left(j 2 \pi x_{i} u / \lambda\right)=\exp \left(\varphi_{A i}\right) .
$$

The $i$ th virtual element $y_{i}$ (hollow) can be obtained from $x_{i}$ with position shift $\Delta x_{i}$ as shown in Fig. 1. With optimized position shift, we can calculate the phase of the virtual element by the phase relationship of adjacent elements. Specially, the position shifts of virtual elements on the edge of the array are constrained to moving inside and the phase of the response $V_{i}$ on the $i$ th virtual can be defined as

$$
\varphi_{V i}= \begin{cases}\varphi_{A i}+\alpha_{i}\left(\varphi_{A i+1}-\varphi_{A i}\right) & \Delta x_{i} \geq 0 \\ \varphi_{A i}+\alpha_{i}\left(\varphi_{A i}-\varphi_{A i-1}\right) & \Delta x_{i}<0\end{cases}
$$

where $\alpha_{i}=\Delta x_{i} / d$ is the position offset factor determined by the distinction between the virtual element and the actual element, and its value range will be discussed hereinafter. From now on, we consider $\alpha_{i} \geq 0$, and the response of the $i$ th virtual element is calculated as

$$
\begin{aligned}
V_{i}(u) & =\exp \left(\varphi_{V i}\right)=\exp \left[\varphi_{A i}+\alpha_{i}\left(\varphi_{A i+1}-\varphi_{A i}\right)\right] \\
& =\exp \left(\varphi_{A i}\right) \exp \left[\alpha_{i}\left(\varphi_{A i+1}-\varphi_{A i}\right)\right] .
\end{aligned}
$$

If the phase offset of adjacent elements exceeds $\pi$, the issue of angle ambiguity will be considered, and here may be grating lobes outside the range of optimization. In this paper, the absolute of phase offset must be less than $\pi$ by setting the spacing of $d$ less than $\lambda / 2$. The response of virtual element can be expressed by the actual element as:

$$
\begin{aligned}
V_{i}(u) & =\left[\exp \left(\varphi_{A i}\right)\right]^{1-\alpha_{i}}\left[\exp \left(\varphi_{A i+1}\right)\right]^{\alpha_{i}} \\
& =\left[A_{i}(u)\right]^{1-\alpha_{i}}\left[A_{i+1}(u)\right]^{\alpha_{i}} .
\end{aligned}
$$

Consequently, the virtual array factor with the steering angle $u$ of signal arrival can be expressed as 


$$
f_{V}(u)=\sum_{i=1}^{M} w_{i} V_{i}(u)
$$

where the phase of $w_{i}$ can be calculated by the steering vector with the signal steering angle $u_{\mathrm{S}}=\sin \left(\theta_{\mathrm{S}}\right)$ and it can be expressed as $w_{i}=\exp \left[-j 2 \pi\left(i+\alpha_{i}\right)(d / \lambda) u_{\mathrm{S}}\right]$ calculated from the new virtual array geometry.

Without loss of generality, the position shifts and the amplitude of $w_{i}$ can be optimized by FPSO in next section. Here, the value range of $\alpha_{i}$ and $\beta_{i}$ will be constrained and optimized synchronously by DPSOA or only the value of $\alpha_{i}$ by DPSM is adjusted to minimize the sidelobe level.

For the real implementation of the proposed DPSM and DPSOA, the best solution of position shifts and optimum amplitudes can be synthesis by high performance computer in advance, and the results can be applied to the signal receiver just by exponentiation operation according to (5) and (6)

As we know, the optimal amplitudes can be realized in the real part of weighting coefficients. When applying the optimal position shifts for array pattern synthesis, the radiation pattern of new elements are calculated by exponent arithmetic, which can be realized in digital signal processor. With the help of such an optimized process, the optimal solution can be realized by digital computation. As a result, the high performance is attractive to synthesize an array by minimizing PSL considering all possible steering directions, respectively, that is, the optimized process has no influence on beam scanning and the minimum PSL of steering direction close to endfire direction cannot restrict other results.

As the objective of optimization is to minimize the sidelobe level of the array pattern by adjusting the parameters of the array, subject to given design specifications and constraints, the fitness function can be defined with the evaluation of the PSL as

$$
\text { fitness }=20 \log _{10}\left(\max \left|\frac{f_{\mathrm{V}}\left(u_{\mathrm{SL}}\right)}{\max f_{\mathrm{V}}(u)}\right|\right)
$$

where $u_{\mathrm{SL}}$ is the spanned angle within the sidelobe band except the range of the mainlobe. The function is then evaluated excluding the mainbeam and the PSL is measured in decibel. In this study, we consider that either the position shift only or with the amplitude of $w_{i}$ will be used to control the minimizing PSL of the array pattern according to the feedback value of (7).

It is well known that array optimization should be organized along a specific trade-off rule between the PSL and the mainlobe width. To simplify this problem, only the minimization of the PSL is considered in the optimization. The mainlobe width is fixed to be within a given range according to the design specifications.

\section{FPSO and Constrained Conditions}

\subsection{FPSO}

PSO, also known as swarm intelligence is a robust stochastic evolutionary computation technique based on the movement of intelligent swarms [20], [21]. What has been proposed in [18] is that the main limitation of PSO is its tendency to converge prematurely at local optima and in [13] the iteration numbers to converge or the number of the fitness function evaluations is an investigative topic.

In this paper, the solution includes position offset factors used as the index and the amplitudes constrained to get a lower Current Taper Ratio (CRT). Case study reveals that choice of mutation probability and mutation step size both have a strong influence on convergence behavior of the swarm. For more mutation probability, there should be large random velocity or large weighting coefficient for velocity update before entering the global optimization range. To enhance searching the precise solution that affects the index of pattern, a small value of velocity and weighting coefficient are important for the absolute best value.

The concept of linearly decreasing inertia weight applied to particle swarm optimization (LPSO) and a method of parameter strategy for PSO was proposed using the overshoot and the peak time of a transition in [19]. In [22], a new adaptive inertia weight adjusting approach is proposed based on Bayesian techniques in PSO, which is used to set up a sound tradeoff between the exploration and exploitation characteristics.

For a large initial searching space and fast convergence in local space with refined solution, we propose nonlinear inertia weight decided by subtriplicate function with feedback taken from the fitness of the best previous position. The feedback function with the inertia weight can be expressed as

$$
\mu_{\mathrm{t}}=\frac{\mu_{\mathrm{max}}+\mu_{\min }}{2}+\frac{\mu_{\mathrm{max}}-\mu_{\mathrm{min}}}{2} \cdot \gamma \cdot \sqrt[3]{F_{\mathrm{FB}}-F_{\mathrm{OP}}-\kappa}
$$

where $\mu_{\mathrm{t}}$ is limited between $\mu_{\max }$ and $\mu_{\min }$, which are the initial and final value of the inertia weight and set to 0.9 and 0.4 , respectively. The terms $\gamma$ and $\kappa$ are independent to control the slope and location of feedback function. $F_{\mathrm{OP}}$ is the expected fitness value of optimal solution and $F_{\mathrm{FB}}$ is the feedback fitness value taken from the best previous position found by the particle.

For different optimization accuracy or kinds of array pattern synthesis and different steering angles, we can set $\gamma$ and $\kappa$ to appropriate values. $F_{\mathrm{OP}}$ will be a prior value through several experiments. In the next section, we describe the performance for different optimal conditions with these sets of parameters. 


\subsection{Constrained Conditions in Optimized Process}

The study that follows has been made on the performance of the DPSM under different conditions by FPSO, where several boundary conditions for position shift and weights are considered. For the signal received from the sensors, the useful signal can be received, which often involves noise item for most applications [24], [25]. $n_{i}$ can be expressed as the spatially and temporally white complex Gaussian noise with zero mean and variance $\sigma_{n}^{2}$ for the $i$ th element [24] and the noise signals are all uncorrelated with useful signal [25]. For (5), noise can be added to it and it can be expressed by one more item as $N_{i}\left(n_{i}, n_{i+1}\right)$, which is the correlation function of $n_{i}$ and $n_{i+1}$. Ulteriorly, it can be expressed as the index relationship with $\alpha_{i}$, that is, $N_{i}\left(n_{i}, n_{i+1}\right)=n_{i}^{1-\alpha_{i}} n_{i+1}{ }^{\alpha_{i}} \quad\left(\right.$ or $\left.N_{i}\left(n_{i}, n_{i-1}\right)=n_{i}^{1+\alpha_{i}} n_{i-1}^{-\alpha_{i}}\right)$. Without loss of generality, when $\alpha_{i}>0$, the noise correlation function about the $i$ th virtual elements can be expressed as

$$
\begin{aligned}
R_{n}^{i, i} & =E\left(N_{i}\left(n_{i}, n_{i+1}\right), N_{i}\left(n_{i}, n_{i+1}\right)\right) \\
& =E\left\{\left(n_{i}^{1-\alpha_{i}} n_{i+1}^{\alpha_{i}}\right)\left(n_{i}^{1-\alpha_{i}} n_{i+1}^{\alpha_{i}}\right)^{\mathrm{H}}\right\}
\end{aligned}
$$

where $E\{\}$ and the superscript $\mathrm{H}$ denote the expectation and complex conjugate transpose, and $R_{n}^{i, i}$ is same with one noise element of the ensemble correlation matrix in [24]. When $\alpha_{i}>0$ and $\alpha_{i+1}<0$, the noise correlation function about the two adjacent virtual elements can be expressed as

$$
\begin{aligned}
R_{n}^{i, i+1} & =E\left(N_{i}\left(n_{i}, n_{i+1}\right), N_{i+1}\left(n_{i+1}, n_{i}\right)\right) \\
& =E\left\{\left(n_{i}^{\alpha_{i}} n_{i+1}{ }^{1-\alpha_{i}}\right)\left(n_{i+1}{ }^{1+\alpha_{i+1}} n_{i}{ }^{-\alpha_{i+1}}\right)^{\mathrm{H}}\right\} .
\end{aligned}
$$

In actual application, for keeping the noise correlation function of actual array consistent with the noise correlation function of virtual array in (9), we need to hold the expectation of the $i$ th virtual element autocorrelation function unchanged. It can also say that $n_{i}^{\alpha_{i}} n_{i+1}{ }^{1-\alpha_{i}}$ or $n_{i}^{1+\alpha_{i}} n_{i-1}^{-\alpha_{i}}$ is kept consistent with the weighted proportions of the actual noise item $n_{i}$, which means that we must select the $\alpha_{i}$ values according to $\left|\alpha_{i}\right|<0.5$ or $\left|\Delta x_{i}\right|<d / 2$. When $\alpha_{i}>0$ and $\alpha_{i+1}<0$, the noise item of the $i$ th and the $(i+1)$ th virtual element are expressed by the $i$ th and the $(i+1)$ th actual element simultaneously. For keeping the cross-correlation feature useful for the two elements and the characteristic of $n_{i}{ }^{\alpha_{i}} n_{i+1}{ }^{1-\alpha_{i}}$ different from $n_{i+1}{ }^{1+\alpha_{i+1}} n_{i}{ }^{-\alpha_{i+1}}$ in (10), now the constraints of interelement position shift is set to $\left(\alpha_{i}-\alpha_{i+1}\right)<0.5,\left(\Delta x_{i}-\Delta x_{i+1}\right)<d / 2$.

For the amplitude of weights constrains, we use the same setting $0 \leq \beta_{i} \leq 2$ in [13]. The CRT is the ratio between the maximum and minimum amplitudes of weight coefficients. The CRT is related to the effects of possible unforeseen occurrences regarding the sensors with the largest weights [23]. Therefore, the value of CRT in designing the pattern is a basis to judge the optimized performance.

\section{Numerical Examples and Results Analysis}

To illustrate the effectiveness of the proposed method, we compare the performance of DPSM and DPSOA with that of improved genetic algorithm (IGA) [13] in beam scanning. For the optimization of linear array, we search the best position shift and optimized amplitude for setting $\varphi=0$ and $\theta_{\mathrm{S}}=0,15,30,45,60$ to synthesize the pattern according to (6). The fitness value of the initial iteration and final iteration has an expectant convergence by FPSO.

In the following examples, the number of linear array elements is $M=10$, which is placed on x-axis with intersensor spacing of $\lambda / 2$. In the FPSO, the initial population is formed by position offset factor of zero and amplitude of one. The population size is set to 50 , and the maximum number of allowable iteration is 100 .

\subsection{Case I: Linear Array with Position Shifts and Uniform Weights}

In this case, only position shift for every element is considered to optimize the pattern. Figure 2 shows the beam pattern in different steering directions, which is the best-case of 10 trials and its PSL values are $-20.40,-18.08$, $-16.41,-14.85$, and $-14.46 \mathrm{~dB}$ given in Tab. 1. Specially, it can be seen from Tab. 1 that the average-case lowest PSL for the DPSM is more $1 \mathrm{~dB}$ lower than that of IGA in case II [13] with the main beams confined to be within $0 \leq \mu \leq 2 / M$. For the IGA in different optimum conditions and different number array elements, the number of fitness function evaluations is invariably higher than 6,704, but for DPSM, it is 5000 in every case.

In [13], considering all possible directions, the minimizing PSL is $11.72 \mathrm{~dB}$ because the minimum PSL achievable when steering direction close to endfire direction is around $-12 \mathrm{~dB}$. For DPSM and DPSOA, there are no compulsory contacts in each steering direction because of digital realization instead of fixed geometry. We can also use the optimal results in Tab. 1 for beam scanning without special limit.

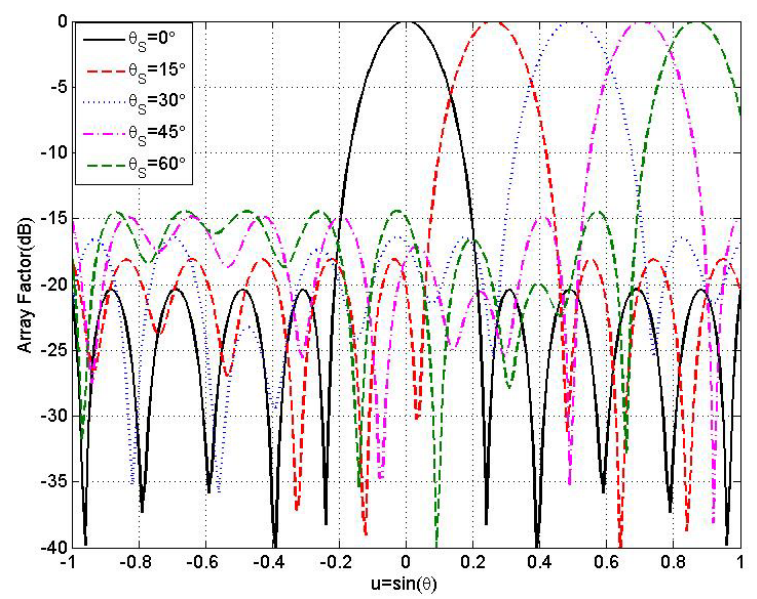

Fig. 2. Resultant beam patterns designed $(M=10)$ with 5 steering directions by DPSM whose PSL is listed in Tab. 1. 


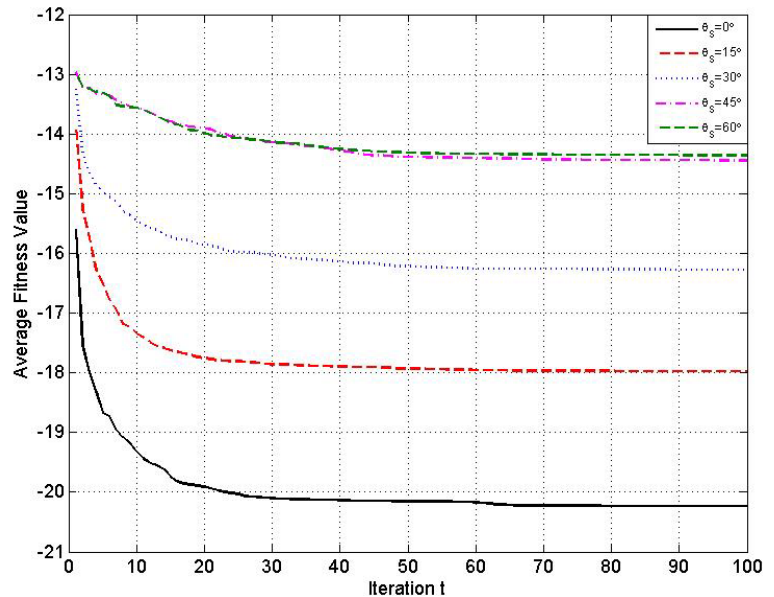

Fig. 3. The mean convergence of fitness values with iteration (averaged over 10 trials) for five steering directions by DPSM.

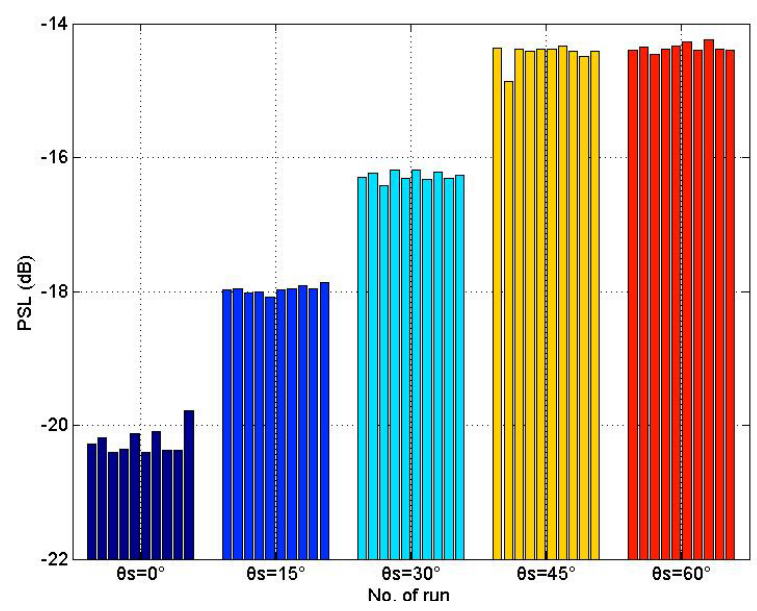

Fig. 4. Resultant lowest PSLs obtained by DPSM in 10 runs.

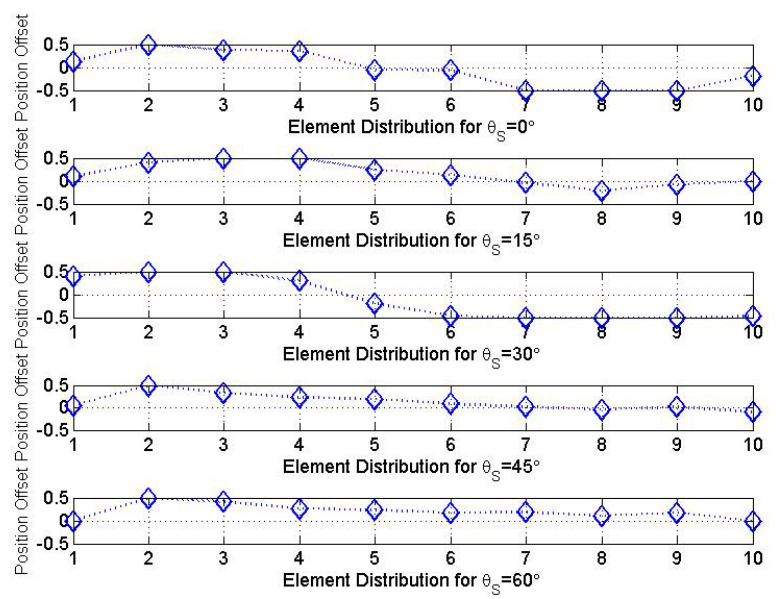

Fig. 5. Best-case sensor position offset factors for five steering directions by DPSM.

In DPSM, the parameters in FPSO are selected as $\gamma=1, \kappa=1.5$ for $\theta_{\mathrm{S}}=0^{\circ}, \gamma=1.25, \kappa=0.55$ for $\theta_{\mathrm{S}}=15^{\circ}$, $\gamma=1.5, \kappa=0.32$ for $\theta_{\mathrm{S}}=30^{\circ}, \gamma=2, \kappa=0.15$ for $\theta_{\mathrm{S}}=45^{\circ}$ and $\theta_{\mathrm{S}}=60^{\circ}$. In different steering directions, $F_{\mathrm{OP}}$ is set to the value of best-case PSL. Figure 3 shows the mean convergence of fitness values with iteration, which has de-

\begin{tabular}{|c|c|c|c|c|c|c|}
\hline $\begin{array}{c}\text { Steering } \\
\text { Direction }\end{array}$ & \multicolumn{2}{|c|}{$\begin{array}{c}\text { Worst-case PSL } \\
\text { (dB) }\end{array}$} & \multicolumn{2}{c|}{$\begin{array}{c}\text { Best-case PSL } \\
\text { (dB) }\end{array}$} & \multicolumn{2}{c|}{$\begin{array}{c}\text { Average-case } \\
\text { PSL (dB) }\end{array}$} \\
\hline Method & IGA & DPSM & IGA & DPSM & IGA & DPSM \\
\hline $0^{\circ}$ & -18.17 & -19.77 & -18.81 & -20.40 & -18.43 & -20.23 \\
\hline $15^{\circ}$ & -15.92 & -17.87 & -16.54 & -18.08 & -16.24 & -17.97 \\
\hline $30^{\circ}$ & -14.47 & -16.18 & -15.06 & -16.41 & -14.88 & -16.27 \\
\hline $45^{\circ}$ & $-12,97$ & -14.34 & -13.64 & -14.85 & -13.27 & -14.44 \\
\hline $60^{\circ}$ & -12.96 & -14.24 & -12.96 & -14.46 & -12.96 & -14.36 \\
\hline
\end{tabular}

Tab. 1. The optimal results for different steering directions through asymmetrical aperture and uniform weights of 10 elements, comparing optimized performance between IGA [13] and DPSM using FPSO.

clined rapidly in the initial iteration and has converged rapidly with a refined solution. The terms $\gamma$ and $\kappa$ are independent to control the slope and location of $\mu_{\mathrm{t}}$. In the five experiments, the value of $\kappa$ is decreasing with increasing of PSL, so an accurate solution can be got in the endfire direction. According to the difference of initial PSL and lowest PSL, we set appropriate value of $\gamma$ to ensure the convergence of FPSO in different directions.

Figure 4 shows resultant lowest PSL in each individual run when the DPSM is employed in the synthesis of five steering directions. Simulation runs also show that the worst-case and average lowest PSL for the IGA are poorer than those from DPSM, repetitively. The variance of those trials can be seen from Fig. 4, and the values have been calculated which are listed in Tab. 1. For the comparing between IGA and DPSM, ten simulation runs can be enough, even though more runs mean more accurate result. Figure 5 shows best-case sensor position offset factors for five steering directions by DPSM.

\subsection{Case II: Linear Array with Position Shifts and Optimum Amplitudes}

In this case, the position shift and amplitude are determined simultaneously using FPSO, and the fitness function evaluations is 5,000 yet. The resultant beam pattern can be obtained with lower PSL in every steering direction, as shown in Fig. 6. The DPSOA is able to achieve the PSL about $0.3 \mathrm{~dB}$ lower than that of the 17-elements aperiodic

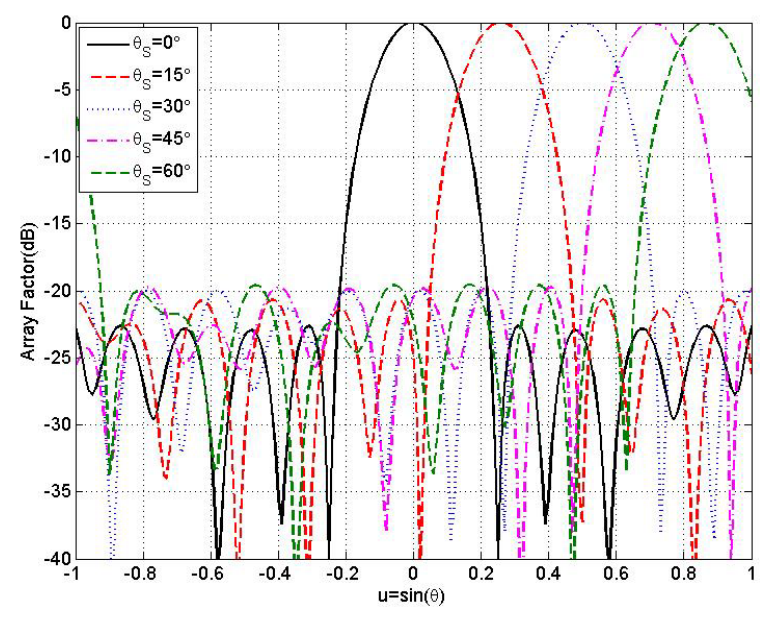

Fig. 6. Resultant beam patterns designed $(M=10)$ with 5 steering directions by DPSOA whose PSL is listed in Tab. 2. 


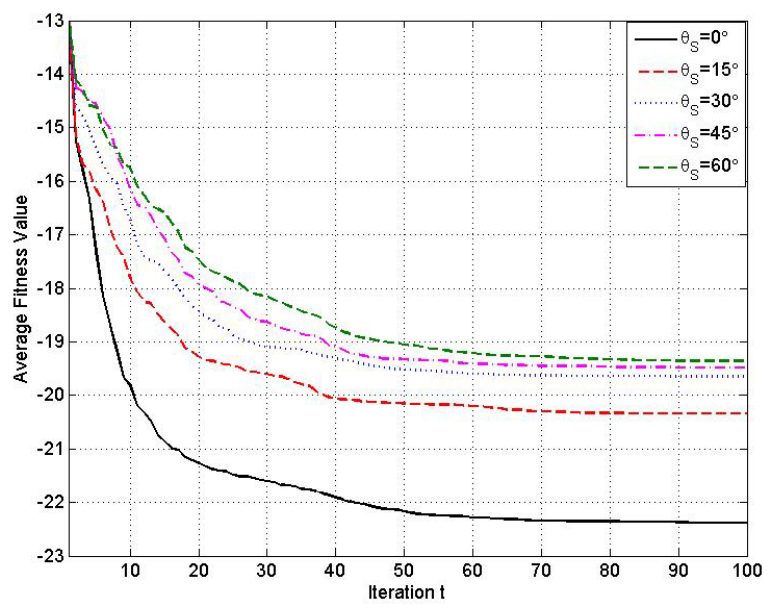

Fig. 7. The mean convergence of fitness value with iteration (averaged over 10 trials) for five steering directions by DPSOA.

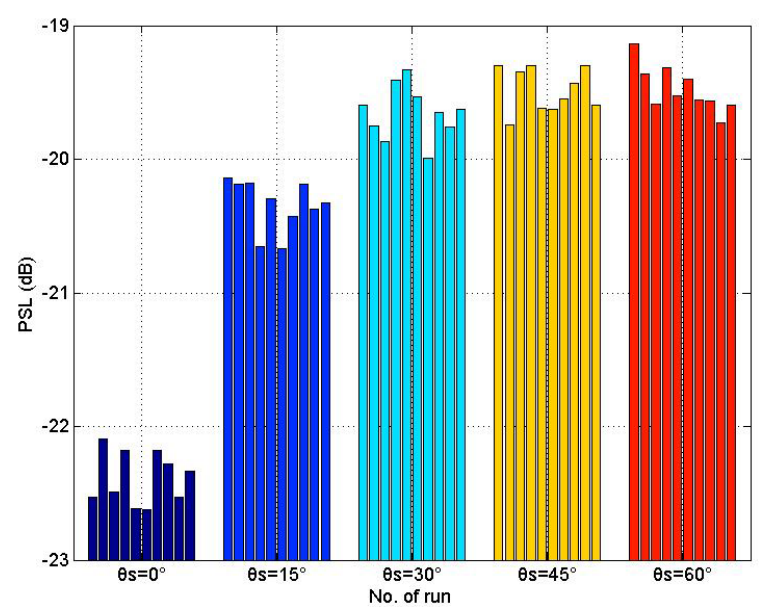

Fig. 8. Resultant lowest PSLs obtained by DPSOA in 10 runs.

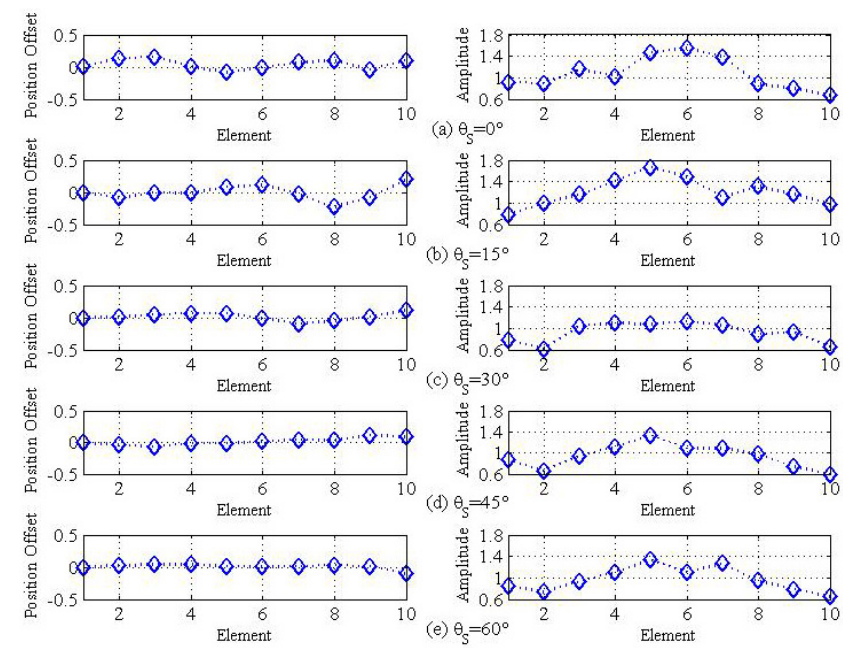

Fig. 9. Best-case sensor position offset factors and optimal amplitudes for five steering directions by DPSOA.

array with asymmetrical aperture and optimum real weights [13] and about $2.2 \mathrm{~dB}$ lower than that of DPSM in the steering direction of $0^{\circ}$. The best-case lower PSL of $-19.72 \mathrm{~dB}$ is more than $5 \mathrm{~dB}$ lower than that of DPSM in the steering direction of $60^{\circ}$. Considering all possible steer-

\begin{tabular}{|c|c|c|c|c|}
\hline $\begin{array}{c}\text { Steering } \\
\text { Direction }\end{array}$ & $\begin{array}{c}\text { Worst-case } \\
\text { PSL (dB) }\end{array}$ & $\begin{array}{c}\text { Best-case } \\
\text { PSL (dB) }\end{array}$ & $\begin{array}{c}\text { Average-case } \\
\text { PSL (dB) }\end{array}$ & $\begin{array}{c}\text { CRT of } \\
\text { Weights(dB) }\end{array}$ \\
\hline $0^{\circ}$ & -22.09 & -22.62 & -22.38 & 3.67 \\
\hline $15^{\circ}$ & -20.13 & -20.66 & -20.34 & 3.26 \\
\hline $30^{\circ}$ & -19.32 & -19.99 & -19.65 & 2.64 \\
\hline $45^{\circ}$ & -19.30 & -19.74 & -19.48 & 3.58 \\
\hline $60^{\circ}$ & -19.13 & -19.72 & -19.47 & 3.16 \\
\hline
\end{tabular}

Tab. 2. The optimal results and CRT values for different steering directions through DPSOM of 10 elements

ing directions, DPSOA can achieve a lower PSL of about $-19 \mathrm{~dB}$, which can be used for beam scanning with a high performance.

For DPSOA, a larger solution space should be considered by FPSO. The convergence of FPSO is more challenging, and the lower PSL can be obtained at the same time. For the higher difference of initial PSL and lowest PSL, the parameters in FPSO are selected as $\gamma=1, \kappa=1.5$ for different steering angles in DPSOA. In Fig. 7, the convergence of fitness value is also consistent with the design of (8). Figure 8 shows resultant lowest PSL in each individual run when the DPSOA is employed in the synthesis of five steering directions. Simulation runs also show that FPSO can ensure the convergence even though the complex solution space. The variance of those trials can be seen from Fig. 8, and the values have been calculated which are listed in Tab. 2.

The design in this case has a CRT of amplitudes lower than $3.67 \mathrm{~dB}$ in all steering directions listed in Tab. 2. Figure 9 shows best-case sensor position offset factors and optimal amplitudes for five steering directions by DPSOA.

\section{Conclusion}

The objective of the proposed DPSM and DPSOA methods is to minimize the PSL and to maintain a desired beam pattern, even as a high performance in beam scanning is achieved through digital realization. Numerical examples show that the DPSM and DPSOA are able to achieve lower PSL with maintaining main beam width. Using FPSO, mean convergence of fitness values with iteration declined rapidly in the initial iteration and converged rapidly with a refined solution and only 5,000 fitness function evaluations are required for the kinds of array synthesis. What is more, the optimized results in all steering directions can be used in beam scanning for its digital realization, which has lower PSL than the IGA [13], especially the steering direction close to endfire direction. In addition, the DPSM and DPSOA can be used for linear array and separable planar array with small computation.

It is not possible to predict the accuracy of the synthesis result, and for the randomness of every optimized procedure, the results cannot exactly be the same. There will be many exciting potential applications of DPSM and DPSOA by properly selecting the optimal algorithm for other geometry arrays such as aperiodic array or conformal array. 


\section{Acknowledgments}

This study was supported in part by the National Natural Science Foundation of China (No. 61271416 and 61301093), the Fundamental Research Funds for the Central Universities (No. 3102014KYJD027), and NPU Foundation for Fundamental Research (No. JCY20130132)

\section{References}

[1] YANG, K., ZHAO, Z. Q., LIU, J. Z., et al. Robust adaptive beamforming using an iterative FFT algorithm. Signal Processing, 2014, vol. 96, no. part B, p. 253-260. DOI: 10.1016/j.sigpro.2013.09.003

[2] EIREY-PÉREZ, R., RODRÍGUEZ-GONZÁLEZ, J. A., ARESPENA, F. J. Synthesis of array radiation pattern footprints using radial stretching, Fourier analysis, and Hankel transformation. IEEE Transactions on Antennas and Propagation, 2012, vol. 60 no. 4, p. 2106-2109. DOI: 10.1109/TAP.2012.2186257

[3] CHATTERJEe, S., CHATTERJEE, S., PODDAR, D. R. Synthesis of linear array using Taylor distribution and Particle Swarm Optimisation. International Journal of Electronics, 2015, vol. 102, no. 3, p. 514-528. DOI: 10.1080/00207217.2014.905993

[4] WANG, X. C., ZHOU, Y. G., WANG, Y. F. An improved antenna array pattern synthesis method using fast Fourier transforms. International Journal of Antennas and Propagation, 2015, vol. 2015,9 p. DOI: $10.1155 / 2015 / 316962$

[5] WANG, X. R., ABOUTANiOS, E., AMIN, M. G. Thinned array beampattern synthesis by iterative soft-thresholding-based optimization algorithms. IEEE Transactions on Antennas and Propagation, 2014, vol. 62, no. 12, p. 6102-6113. DOI: 10.1109/TAP.2014.2364048

[6] ZHANG, F. G., JIA, W. M., YAO, M. L. Linear aperiodic array synthesis using differential evolution algorithm. IEEE Antennas and Wireless Propagation Letters, 2013, vol. 12, p. 797-800. DOI: 10.1109/LAWP.2013.2270930

[7] SHARAQA, A., DIB, N. Position-only side lobe reduction of a uniformly excited elliptical antenna array using evolutionary algorithms. IET Microwaves Antennas and Propagation, 2013, vol. 7, no. 6, p. 452-457. DOI: 10.1049/iet-map.2012.0541

[8] CEN, L., SER, W., YU, Z. L., et al. Linear sparse array synthesis with minimum number of sensors. IEEE Transactions on Antennas and Propagation, 2010, vol. 58, no. 3, p. 720-726, DOI: 10.1109/TAP.2009.2039292

[9] ABU-AL-NADI, D. I., ISMAIL, T. H., AL-TOUS, H., et al. Design of linear phased array for interference suppression using array polynomial method and particle swarm optimization. Wireless Personal Communications, 2012, vol. 63, no. 2, p. 501-513. DOI: $10.1007 / \mathrm{s} 11277-010-0146-\mathrm{x}$

[10] ISMAIL, T. H., HAMICI, Z. M. Array pattern synthesis using digital phase control by quantized particle swarm optimization. IEEE Transactions on Antennas and Propagation, 2010, vol. 58, no. 6 , p. 2142-2145. DOI: 10.1109 /TAP.2010.2046853

[11] ELKAMCHOUCHI, H. M., HASSAN, M. M. Array pattern synthesis approach using a genetic algorithm. IET Microwaves Antennas and Propagation, 2014 vol. 8, no. 14, p. 1236-1240. DOI: $10.1049 /$ iet-map.2013.0718

[12] BEVELACQUA, P. J., BALANIS, C. A. Minimum sidelobe levels for linear arrays. IEEE Transactions on Antennas and Propagation, 2007, vol. 55, no. 12 , p. 3442-3449. DOI: 10.1109/TAP.2007.910490
[13] CEN, L., YU, Z. L., SER, W., et al. Linear aperiodic array synthesis using an improved genetic algorithm. IEEE Transactions on Antennas and Propagation, 2012, vol. 60, no. 2, p. 895-902. DOI: 10.1109/TAP.2011.2173111

[14] VASKELAINEN, L. I. Virtual array synthesis method for planar array antennas. IEEE Transactions on Antennas and Propagation, 1998, vol. 46, no. 3, p. 391-396. DOI: 10.1109/8.662658

[15] TRUCCO, A. Synthesizing asymmetric beam patterns. IEEE Journal of Oceanic Engineering, 2000, vol. 25, no. 3, p. 347-350. DOI: $10.1109 / 48.855383$

[16] KURUP, D. G., HIMDI, M., RYDBERG, A. Synthesis of uniform amplitude unequally spaced antenna arrays using the differential evolution algorithm. IEEE Transactions on Antennas and Propagation, 2003, vol. 51, no. 9, p. 2210-2217. DOI: 10.1109/TAP.2003.816361

[17] JIN, N., RAHMAT-SAMII, Y. Hybrid real-binary particle swarm optimization (HPSO) in engineering electromagnetics. IEEE Transactions on Antennas and Propagation, 2010, vol. 58, no. 12, p. 3786-3794. DOI: 10.1109/TAP.2010.2078477

[18] BHATTACHARYA, R., BHATTACHARYYA, T. K., GARG, R. Position mutated hierarchical particle swarm optimization and its application in synthesis of unequally spaced antenna arrays. IEEE Transactions on Antennas and Propagation, 2012, vol.60, no. 7, p. 3174-3181. DOI: 10.1109/TAP.2012.2196917

[19] ZHANG, W., MA, D., WEI, J. J., et al. A parameter selection strategy for particle swarm optimization based on particle positions. Expert Systems with Applications, 2014, vol. 41, no. 7, p. 3576 to 3584. DOI: 10.1016/j.eswa.2013.10.061

[20] BERA, R., MANDAL, D., KAR, R., et al. Application of particle swarm optimization technique in hexagonal and concentric hexagonal antenna array for side lobe level reduction. Advances in Intelligent Systems and Computing, 2015, vol. 343, p. 333-347. DOI: $10.1007 / 978-81-322-2268-2 \_36$

[21] MODIRI A., KIASALEH, K. Modification of real-number and binary PSO algorithms for accelerated convergence. IEEE Transactions on Antennas and Propagation, 2011, vol. 59, no. 1, p. 214-224. DOI: 10.1109/TAP.2010.2090460

[22] ZHANG, L. M., TANG, Y. G., HUA, C. C., et al. A new particle swarm optimization algorithm with adaptive inertia weight based on Bayesian techniques. Applied Soft Computing, 2015, vol. 28, p. 138-149. DOI: $10.1016 /$ j.asoc.2014.11.018

[23] TRUCCO A., MURINO, V. Stochastic optimization of linear sparse arrays. IEEE Journal of Oceanic Engineering, 1999, vol. 24 no. 3, p. 291-299. DOI: 10.1109/48.775291

[24] CHANG, J. C. A robust adaptive array beamformer using particle swarm optimization for space-time code division multiple access systems. Information Sciences, 2014, vol. 278, p. 174-186. DOI: 10.1016/j.ins.2014.03.036

[25] KHABBAZIBASMENJ, A., VOROBYOV, S. A., HASSANIEN, A. Robust adaptive beamforming based on steering vector estimation with as little as possible prior information. IEEE Transactions on Signal Processing, 2012, vol. 60, no. 6, p. 2974-2987. DOI: 10.1109/TSP.2012.2189389

\section{About the Authors ...}

Chuang HAN (corresponding author) was born in Hebei, China, in 1989. He received the B.Sc. and M.Sc. degrees in Electronic Engineering from the Northwestern Polytechnical University in 2012 and 2015, respectively. Currently, he is working on a Ph.D. degree in the Northwestern Polytechnical University. His recent research interests include 
array signal process, antenna analysis and synthesis, satellite communication systems, and satellite navigation systems.

Ling WANG was born in Guizhou, China, in 1978. He received the B.Sc., M.Sc., and Ph.D. degrees from Xidian University, Xi'an, China, in 1999, 2002 and 2004, respectively. From 2004 to 2007, he worked at Siemens Academy of wireless communication unit and Nokia Siemens Networks research center. Since 2007, he has been with the School of Electronic and Information, Northwestern Polytechnical University. His current research interests include B3G/4G mobile communications; vehicle tracking, telemetry, and command (TT\&C); anti-jamming for communications and navigation systems; cognitive radio. 